\title{
A Generalization of Hermite Polynomials
}

\author{
G. M. Habibullah \\ National College of Business Administration \& Economics \\ Gulberg-III, Lahore, Pakistan \\ mustafa1941@yahoo.com
}

Abdul Shakoor

National College of Business Administration \& Economics

Gulberg-III, Lahore, Pakistan

chshakoor@gmail.com

Copyright (c) 2013 G. M. Habibullah and Abdul Shakoor. This is an open access article distributed under the Creative Commons Attribution License, which permits unrestricted use, distribution, and reproduction in any medium, provided the original work is properly cited.

\begin{abstract}
The intended objective of this paper is to extend the Hermite polynomials based on hypergeometric functions and to prove basic properties of the extended Hermite polynomials.

Mathematics Subject Classification: 33C45, 05A15, $11 \mathrm{~B} 37$.

Keywords: Orthogonal polynomials, Hermite polynomials, Hypergeometric functions, Generalized hypergeometric functions.

\section{Introduction}

Hermite Polynomials and its applications have been studied for long and still attract attention. One can refer a long list of books and journals for advanced knowledge of Hermite polynomials and its extensions, for example [7] and [6], for books and [2], [4] , [5], [7], [8], [9], [10] and [11] for journals. Based on a generalized hypergeometric function, we introduce here a generalization of the Hermite polynomials that provide natural extensions of basic results involving the Hermite polynomials as a study of the Laguerre polynomials in [3].
\end{abstract}


For a positive integer $p$, the set $\left\{S_{p, n}(x)\right\}$ generated by the function $G(x, t)=\exp \left(p x t-t^{p}\right)$ is to be known as the generalized Hermite polynomial set. Note that for $p=2$, it reduces to the known generating function for the Hermite polynomials. We first deduce an explicit expression for this generalized Hermite polynomials.

\section{Theorem 1:}

For a non-negative integer $n$ and a positive integer $p$, we have

$$
S_{p, n}(x)=\sum_{k=0}^{\left[\frac{n}{p}\right]} \frac{(-1)^{k} n !(p x)^{n-p k}}{k !(n-p k) !} .
$$

Proof:

By Considering

$$
\sum_{n=0}^{\infty} \frac{S_{p, n}(x) t^{n}}{n !}=e^{\left(p x t-t^{p}\right)},
$$

note that

$$
\begin{aligned}
e^{\left(p x t-t^{p}\right)} & =\left(\sum_{n=0}^{\infty} \frac{(p x t)^{n}}{n !}\right)\left(\sum_{k=0}^{\infty} \frac{\left(-t^{p}\right)^{k}}{k !}\right) \\
& =\sum_{n=0}^{\infty} \sum_{k=0}^{\infty} \frac{(-1)^{k}}{k !} \frac{(p x)^{n} t^{n+p k}}{n !}
\end{aligned}
$$

A use of a variation of Lemma 11 pp. 57 of [6] with $\frac{n}{p}$ in place of $\frac{n}{2}$ leads to

$$
\sum_{n=0}^{\infty} \frac{S_{p, n}(x) t^{n}}{n !}=\sum_{n=0}^{\infty} \sum_{k=0}^{\left[\frac{n}{p}\right]} \frac{(-1)^{k}(p x)^{n-p k} t^{n}}{k !(n-p k) !},
$$

which implies that

$$
S_{p, n}(x)=\sum_{k=0}^{\left[\frac{n}{p}\right]} \frac{(-1)^{k} n !(p x)^{n-p k}}{k !(n-p k) !} .
$$


We now determine a few recurrence relations for the generalized Hermite polynomials.

\section{Theorem 2:}

For all finite $x, t$, a positive integer $p$ and a non-negative integer $n$,

(i) $x S_{p, n}^{\prime}(x)-n S_{p, n}(x)=n(n-1)(n-2) \cdots(n-p+2) S_{p, n-p+1}^{\prime}(x),(1.2)$

(ii) $n p(n-p+2) S_{p, n}(x)$

$$
=p x(n-p+2) S_{p, n}^{\prime}(x)-n(n-1)(n-2) \cdots(n-p+1) S_{p, n-p+2}^{\prime \prime}(x) .
$$

\section{Proof:}

$$
\text { Consider } F=G\left(p x t-t^{p}\right)=\sum_{n=0}^{\infty} f_{n}(x) t^{n} \text {. }
$$

Taking partial derivatives of $F$ w.r.t $x$ and $t$, we have

$$
\frac{\partial F}{\partial x}=p t G^{\prime}, \frac{\partial F}{\partial t}=\left(p x-p t^{p-1}\right) G^{\prime} .
$$

Also, $\quad \sum_{n=0}^{\infty} x f_{n}^{\prime}(x) t^{n}-\sum_{n=0}^{\infty} f_{n}^{\prime}(x) t^{p+n-1}-\sum_{n=0}^{\infty} n f_{n}(x) t^{n}=0$.

These relations give rise to

$$
\left(x-t^{p-1}\right) \frac{\partial F}{\partial x}-t \frac{\partial F}{\partial t}=0,
$$

and consequently

$$
x f_{n}^{\prime}(x)-n f_{n}(x)=f_{n-p+1}^{\prime}(x) .
$$

Since by taking $G=e^{p x t-t^{p}}, f_{n}(x)=\frac{S_{p, n}(x)}{n !}$, we finally get

$$
x S_{p, n}^{\prime}(x)-n S_{p, n}(x)=n(n-1)(n-2) \cdots(n-p+2) S_{p, n-p+1}^{\prime}(x) .
$$

Similarly, we can prove (ii).

\section{Theorem 3:}

For any real number $c$ and a positive integer $p$, we have 
(i) $\begin{aligned} \sum_{n=0}^{\infty} & \frac{(c)_{n} S_{p, n}(x) t^{n}}{n !}=(1-p x t)^{-c} \times \\ & { }_{p} F_{0}\left(\frac{c}{p}, \frac{c+1}{p}, \frac{c+2}{p}, \ldots, \frac{c+p-1}{p} ;-;(-1)\left(\frac{p t}{1-p x t}\right)^{p}\right),\end{aligned}$

(ii) $S_{p, n}(x)=(p x)^{n}{ }_{p} F_{0}\left(\frac{-n}{p}, \frac{-n+1}{p}, \frac{-n+2}{p}, \ldots, \frac{-n+p-1}{p} ;-; \frac{-1}{x^{p}}\right)$,

(iii) $x^{n}=\sum_{k=0}^{\left[\frac{n}{p}\right]} \frac{n ! S_{p, n-p k}(x)}{p^{n}(n-p k) ! k !}$.

Proof:

Note that

$$
\begin{aligned}
\sum_{n=0}^{\infty} \frac{(c)_{n} S_{p, n}(x) t^{n}}{n !} & =\sum_{n=0}^{\infty} \sum_{k=0}^{\left[\frac{n}{p}\right]} \frac{(-1)^{k}(c)_{n}(p x)^{n-p k}}{k !(n-p k) !} t^{n} \\
& =\sum_{n=0}^{\infty} \sum_{k=0}^{\infty} \frac{(-1)^{k}(c)_{n+p k}(p x)^{n} t^{n+p k}}{k ! n !} \\
& =\sum_{k=0}^{\infty}\left(\sum_{n=0}^{\infty} \frac{(c+p k)_{n}(p x t)^{n}}{n !}\right)\left(\frac{(-1)^{k}(c)_{p k} t^{p k}}{k !}\right),
\end{aligned}
$$

so that

$$
\sum_{n=0}^{\infty} \frac{(c)_{n} S_{p, n}(x) t^{n}}{n !}=\sum_{k=0}^{\infty} \frac{(-1)^{k}(c)_{p k} t^{p k}}{k !(1-p x t)^{c+p k}},
$$

which implies that

$$
\begin{aligned}
& \sum_{n=0}^{\infty} \frac{(c)_{n} S_{p, n}(x) t^{n}}{n !}=(1-p x t)^{-c} \times \\
&{ }_{p} F_{0}\left(\frac{c}{p}, \frac{c+1}{p}, \frac{c+2}{p}, \ldots, \frac{c+p-1}{p} ;-;(-1)\left(\frac{p t}{1-p x t}\right)^{p}\right) .
\end{aligned}
$$


Starting with $S_{p, n}(x)=(p x)^{n} \sum_{k=0}^{\left[\frac{n}{p}\right]} \frac{n !(-1)^{k}}{(n-p k) !} \frac{(p)^{-p k}(x)^{-p k}}{k !}$, and $e^{p x t}=e^{t^{p}} \sum_{n=0}^{\infty} \frac{S_{p, n}(x) t^{n}}{n !}$, we can prove (ii) and (iii).

Following traditional theory, we can prove orthogonality, integrals and expansions involving the Hermite polynomials and its relations with other polynomials. We can also consider $q$-Hermite polynomials and prove corresponding results.

\section{References}

[1] A. Altin and E. Erkus, On a multivariable extension of the LagrangeHermite polynomials, Integral Transforms and Special Functions, (2006), 1476-8291.

[2] A. J. Duran, Rodrigue's formulas for orthogonal matrix polynomials satisfying higher-order differential equations. Experimental Mathematics, 20 (2011), 15-24.

[3] A. Khan and G. M. Habibullah, Extended Laguerre polynomials. Int. J. Contemp. Math. Sci., 22 (2012), 1089-1094.

[4] C. Berg and A. Ruffing, Generalized $q$-Hermite polynomials. Comm. Math. Phys., 223 (2001), 29-46.

[5] C. Kaanoglu and M. A. Ozarslan, Some properties of generalized multiple Hermite polynomials. J. Comp. Appl. Math., 235(2011), 4878-4887.

[6] E. D. Rainville, Special Functions, The Macmillan Company. New York, 1960.

[7] G. Andrews, R. Askey and R. Roy. Special Functions, Cambridge University Press, 1999.

[8] H. Chaggara and W. Koepf, On linearization and connection coefficients for generalized Hermite polynomials, J. Comp. Appl. Math., 236 (2011), 65-73. 
[9] K. Y. Chen and H. M. Srivastava, A limit relationship between Laguerre and Hermite polynomials. Integral Transforms and Special Functions, 16 (2005), 75-80.

[10] R. S. Batahan, A new extension of Hermite matrix polynomials and its applications. Linear Algebra and its Applications, 419 (2006), 82-92.

[11] S. B. Trickovic and M. S. Stankovic, A new approach to the orthogonality of the Laguerre and Hermite polynomials. Integral Transforms and Special Functions, 17 (2006), 661-672.

Received: January 11, 2013 